OPEN ACCESS

Edited by:

Youri I. Pavlov,

University of Nebraska Medical Center,

United States

Reviewed by:

Prashanth N. Suravajhala,

Birla Institute of Scientific Research,

India

Wei Xu,

Texas A\&M University Corpus Christi,

United States

*Correspondence:

Zhenghong Sui

suizhengh@ouc.edu.cn

Specialty section:

This article was submitted to Genomic Assay Technology,

a section of the journal

Frontiers in Genetics

Received: 13 May 2019 Accepted: 14 November 2019 Published: 06 December 2019

Citation:

Hu Y, Sui Z, Zhou W, Wang J, Jiang $M$, Chen $H$, Zhang J, Zhang W, Feng $X$, Lei Y, Xiao B and Guo P (2019) Heterozygous Single Nucleotide

Polymorphic Loci in Haploid

Gametophytes of Gracilariopsis lemaneiformis (Rhodophyta).

Front. Genet. 10:1256

doi: 10.3389/fgene.2019.01256

\section{Heterozygous Single Nucleotide Polymorphic Loci in Haploid Gametophytes of Gracilariopsis lemaneiformis (Rhodophyta)}

\author{
Yiyi Hu, Zhenghong Sui ${ }^{*}$, Wei Zhou, Jinguo Wang, Minjie Jiang, Haihong Chen, \\ Jingyu Zhang, Wei Zhang, Xiaoqing Feng, Yuehua Lei, Baoheng Xiao and Peng Guo
}

Key Laboratory of Marine Genetics and Breeding (Ocean Univeristy of China), Ministry of Education, Qingdao, China

Gracilariopsis lemaneiformis is an important commercial macroalga. Whole-genome resequencing was conducted to identify single nucleotide polymorphisms (SNPs) in parental gametophytes and $60 \mathrm{~F}_{1}$ gametophytes of Gp. lemaneiformis in this study, and 9,989 SNPs located in nonrepetitive sequences were obtained. Among these SNPs, 92.02\% of loci were identified as having a heterozygous genotype in at least one gametophyte, and $48.07 \%$ of loci had identical heterozygous genotypes in the 62 gametophytes. For each gametophyte, the proportions of homozygous and heterozygous loci ranged between 13.74 and $21.61 \%$ (mean of $17.04 \%$ ) and between 66.36 and $83.59 \%$ (mean of $77.12 \%$ ), respectively. The remainder were missing loci, representing an average $5.84 \%$. Sources of heterozygous SNPs were free of exogenous DNA contamination, cross contamination among individuals, plastid and mitochondrial sequences, chimeras of different thallus parts or different cells, and repetitive sequences. Genotypes of heterozygous SNPs were verified by Sanger sequencing of PCR products and monoclones. Duplications of chromosomal rearrangements in the genome of Gp. lemaneiformis might explain the presence of heterozygous SNPs in haploid gametophytes.

Keywords: Gracilariopsis lemaneiformis, haploid gametophyte, single nucleotide polymorphism, heterozygous genotype, whole-genome resequencing

\section{INTRODUCTION}

Gracilariopsis lemaneiformis (Bory de Saint-Vincent) E. Y. Dawson, Acleto \& Foldvik is a commercially important macroalga that is mainly utilized for agar extraction and abalone aquaculture (Santelices and Doty, 1989; Mchugh, 1991). The high content of good quality agar produced by Gp. lemaneiformis represents $53 \%$ of the annual agar output worldwide (Mchugh, 1991). Gp. lemaneiformis is rich in glutamic acid and taurine, which can enhance the flavor of abalone and promote its feed intake (Chopin et al., 2001; Fei, 2004; Neori et al., 2004; Lu et al., 2011). In addition, Gp. lemaneiformis can effectively reduce the contents of $\mathrm{N}$ and $\mathrm{P}$ in the seawater and plays a role in bioremediation (Fei, 2004).

Gp. lemaneiformis is an ideal material for genetic research. Its whole life history can be completed in a laboratory culture (Plastino and de Oliveira, 1988). Mature tetrasporophytes (2n) 
produce tetraspores (n) through meiosis, and then tetraspores are released and develop into male and female gametophytes (n) (Engel et al., 2010). After fertilization, the zygotes develop into carposporophytes $(2 n)$, which release carpospores $(2 n)$ through mitosis (Guzmán-Urióstegui et al., 2002). Subsequently, carpospores develop into tetrasporophytes (Yamamoto and Sasaki, 1988). The reproductive modes of Gp. lemaneiformis include both sexual and asexual reproduction. In recent years, genetic studies on Gp. lemaneiformis have focused on genetic diversity analyses (Wang et al., 2007; Pang et al., 2010; Ding et al., 2012; Hu et al., 2018), functional gene studies (Liu et al., 2016; Liu et al., 2018; Hu et al., 2019), mutation research (Zhang and van der Meer, 2011; Fu et al., 2014), mitochondrial genome (Zhang et al., 2012), chloroplast genome (Du et al., 2016) and genomic studies (Zhou et al., 2013; Sun et al., 2018)

Currently, Gp. Lemaneiformis is broadly cultivated in Guangdong, Fujian, Zhejiang, Shandong, and Liaoning Provinces, representing the second most cultivated seaweed in China. Screening for new strains and developing large-scale industrialized cultivation methods are of great significance for the use of this species. Molecular marker-assisted breeding technology can be used for mapping important quantitative trait loci (QTL), allowing the selection of a trait by analyzing molecular markers that are closely linked to the target trait's loci. It is an effective way to accelerate the breeding process (Ribaut and Hoisington, 1998). Genetic linkage maps play a fundamental role in QTL localization by providing selectable markers for traits selection (Li et al., 2015; Lu et al., 2018; Raman et al., 2018). Due to their relative abundance, high polymorphism rate, stable inheritance, and wide genomic distributions, single nucleotide polymorphism (SNP) markers have been widely applied in molecular marker assisted breeding (Berger et al., 2001; Wicks et al., 2001; Stickney et al., 2002; Chen et al., 2008). The development of SNP markers for breeding have never been conducted in Gp. lemaneiformis. With the advent of next-generation sequencing which provide rapid and low-cost methods for genotyping large populations, SNP markers can be obtained using reduced-representation sequencing (Baird et al., 2008; Kerstens et al., 2009; Wang et al., 2012; Poland et al., 2012; Fu et al., 2016) and whole-genome resequencing (Xia et al., 2009; Huang et al., 2009; Lam et al., 2010; Cao et al., 2011).

Because of the industrial importance of the species and the usefulness of SNP markers in species breeding, SNPs were exploited in the parental gametophytes and $60 \mathrm{~F}_{1}$ gametophytes of Gp. lemaneiformis in this study. If the $\mathrm{F}_{1}$ tetrasporophyte $(2 \mathrm{n})$ is heterozygous at one SNP locus, $\mathrm{F}_{1}$ gametophytes $(\mathrm{n})$ should be homozygous and the segregation ratios of the two genotypes should be $1: 1$ at this locus. However, we identified abundant heterozygous SNP loci in gametophytes, and we intend to investigate where the heterozygous SNP loci originated. This study promotes investigating the genomic characteristics of Gp. lemaneiformis, which will be of great importance in guiding studies on the genomic evolution of marine algae.

\section{MATERIALS AND METHODS}

\section{Construction of Mapping Population}

The life history of Gp. lemaneiformis includes a gametophyte phase, which is equal to the haploid population, and it can be used for genetic linkage mapping. In this study, the female gametophyte $\$ 6$ and male gametophyte 09 were produced from Cultivar Lulong No. 1, which is tetrasporophyte strain. $\mathrm{F}_{1}$ tetrasporophytes were produced from a cross between $\$ 6$ and §9. Then, mature $F_{1}$ tetrasporophytes gave rise to tetraspores, which subsequently developed into $F_{1}$ gametophytes. Wholegenome resequencing was carried out for the two parental gametophytes and $60 \mathrm{~F}_{1}$ gametophytes.

\section{Whole-Genome Resequencing Protocol}

Genomic DNA was extracted using a Plant Genomic DNA Kit (Tiangen Biotech, Beijing, China) following the manufacturer's instructions, and at least $1 \mu \mathrm{g}$ qualified genomic DNA was used for constructing a whole-genome resequencing library. Genomic DNA was sheared randomly into fragments, and 300-500-bp fragments were purified using gel electrophoresis. The sequencing library was prepared using a TruSeq ${ }^{\mathrm{TM}}$ DNA Sample Prep Kit and a TruSeq PE Cluster Kit. The prepared library was sequenced with paired end reads $(2 \times 150 \mathrm{bp})$ on an Illumina HiSeq 4000 sequencing platform. The whole-genome resequencing data of 62 gametophytes have been deposited in Short Read Archive (SRA) database with Project number PRJNA574029, under the accession number SRP223151.

Raw sequence reads were processed using custom PERL scripts to separate them into individual fastq format files based on the barcode sequence of each sample. Contamination reads, like those containing adaptors or primers, were identified using SeqPrep (https://github.com/jstjohn/SeqPrep) with the following parameters: "'-q 20 -L 75 -B AGATCGGAAGAGCGTCGTGT -A AGATCGGAAGAGCACACGTC.” Sickle (https://github. com/najoshi/sickle) was applied to perform read data trimming with default parameters to produce clean data (high quality data) in this study. The high quality sequencing reads were aligned to the reference genome sequence using BWA (http://bio-bwa .sourceforge.net/) software in "bwa aln" mode. The genome survey sequencing of Gp. lemaneiformis had been conducted previously (Zhou et al., 2013), and the assembled scaffolds were used as reference genome sequences in this study. After removing PCR-duplication reads using SAMtools (http://sa mtools.sourceforge.net/) software, the sequencing depth and coverage were calculated based on alignments performed by custom PERL scripts.

\section{SNP Calling}

The valid BAM file was used to detect SNPs using the GATK "UnifiedGenotyper" function (http://www.broadinstitute.org/ga $\mathrm{tk} /$ ). Then, variant call format (VCF) files were generated by qua lity filtering (VariantFiltration with parameters: QD $<2.0|| \mathrm{FS}>$ 60.0|| $\mathrm{MQ}<40.0$ || SOR > 10.0). Furthermore, VCF files were filtered using VCFtools (version 0.1.11; parameters: -minQ 20 $\operatorname{minDP} 4)$. 


\section{Verification of Heterozygous SNP Loci}

The SNP loci that had identical heterozygous genotypes in the two parents, were selected to determine whether they belonged to exogenous DNA contamination. Sequences of $400 \mathrm{bp}$ in which a heterozygous SNP locus was located were obtained from reference genome sequences, and served as target sequences. BLASTN in NCBI with default parameters was used for sequence annotation. The SNP loci that had a genotype that was homozygous (or missing) in one parent but heterozygous in the other, were suspected to result from cytoplasmic inheritance. Sequences of $400 \mathrm{bp}$ containing the target SNP were aligned to the complete plastid and mitochondrial sequences.

SNP primers were designed using Primer 6.0 and synthesized by Sangon Biotech (Shanghai, China). The reaction solution for PCR amplification contained 20 ng genomic DNA, 1× PCR buffer, $1.5 \mathrm{~mm} \mathrm{Mg}{ }^{2+}, 0.2 \mathrm{~mm}$ dNTPs, $0.5 \mu \mathrm{m}$ of each primer, 0.5 U Taq DNA polymerase (Fermentas, Burlington, Canada) and autoclaved distilled water to a total volume of $20 \mu \mathrm{l}$. The PCR program performed was as follows: initial denaturation at $94^{\circ} \mathrm{C}$ for $5 \mathrm{~min}$; 35 cycles of denaturation for $1 \mathrm{~min}$ at $94^{\circ} \mathrm{C}$, annealing for $1 \mathrm{~min}$ at $60^{\circ} \mathrm{C}$ and extension for $1 \mathrm{~min}$ at $72^{\circ} \mathrm{C}$; a final extension step for $5 \mathrm{~min}$ at $72^{\circ} \mathrm{C}$. Sanger sequencing of PCR products and monoclones were completed by TsingKe Biological Technology (Qingdao, China)

\section{Chimera Testing}

To determine whether heterozygous SNPs resulted from chimerism, a single gametophyte of Gp. lemaneiformis was cut from the base to the top, obtaining $221 \mathrm{~cm}$ segments. The segments included nine representative parts: base, middle part, and tip of primary/secondary branch; junctions of primary and secondary branches; junctions of secondary and tertiary branches; tertiary branches. The genomic DNA of every segment was extracted and amplified using primers for SNP locus scaffold1837_730, scaffold7339_502 and scaffold7339_533, and then, the PCR products were analyzed by Sanger sequencing.

\section{SNP Detection in Single Cells}

A gametophyte thallus was used to produce a cell suspension by grinding in liquid nitrogen and filtering. Then, single cells were isolated in a glass capillary tube under a microscope. Genomic DNA of each cell was extracted and amplified using a Single Cell Whole Genome Amplification Kit (Yikon Genomics, Suzhou, China) following the manufacturer's instructions. Then, heterozygous SNP loci were identified using PCR amplification and Sanger sequencing.

\section{RESULTS}

\section{Development of SNPs Using Whole- Genome Resequencing Technology}

The statistical data of the whole-genome resequencing are listed in Table S1. The total data for the female gametophyte parent, male gametophyte parent and the mean of the $F_{1}$ gametophytes were $1.21,1.36$ and $1.62 \mathrm{~Gb}$, respectively. The GC content range per individual was between 45.03 and $52.53 \%$, with a mean of $48.92 \%$, and all three values were in the normal range. The mean Q20 value was greater than 95\%, and the mean Q30 value was greater than $91 \%$, revealing the high quality of sequencing. The depth of the female, male gametophyte parents, and the mean of the $\mathrm{F}_{1}$ gametophytes, were $12 \times, 14 \times$ and $16 \times$, respectively. Each gametophyte was sequenced to an average $80.15 \%$ coverage of the genome. The base distribution and base quality distribution of whole-genome resequencing data were shown in Figures S1 and $\mathbf{S 2}$.

The high quality sequencing reads of each individual were aligned to the reference genome sequences, and the mapping results are displayed in Table S2. The mapping rate of the female parent was $84.35 \%$ and that of the male parent was $49.15 \%$. The percentage of mapped reads among the $60 \mathrm{~F}_{1}$ gametophytes was between 30.20 and $83.75 \%$, with an average of $57.33 \%$. The differences in the mapping rates among individuals might be caused by exogenous DNA contamination or unprovided reference genome sequences of Gp. lemaneiformis.

As a result, the miss rate of SNP genotypes was less than $15 \%$ in 62 gametophytes, and 46,499 SNPs were identified, representing $1.83 \%$ homozygous loci (no homozygous genotypes occurred in the 62 gametophytes) and $98.17 \%$ heterozygous loci (heterozygous genotypes occurred in at least one individual). Further statistical analyses revealed that 22,640 SNP loci had identical heterozygous genotypes in the 62 gametophytes, accounting for $48.69 \%$ of the total SNPs.

\section{Discarding SNPs Located in Repetitive Sequences}

The annotation of repetitive sequences had been conducted previously (Zhou et al., 2013). SNP loci that were located in repetitive sequences were discarded, and 9,989 SNP markers remained. In these SNPs, the number of loci that were identified as having a heterozygous genotype in at least one individual accounted for $92.02 \%$, and $48.07 \%$ loci had identical heterozygous genotypes in the 62 gametophytes. In addition, the number of homozygous SNPs with different genotypes in the two parents was only 25, and these loci did not have 1:1 segregation ratios in the $60 \mathrm{~F}_{1}$ gametophytes. Genotyping results of representative SNP loci in two parents and partial gametophytes are displayed in Table 1. Complete genotype information for the 9,989 SNPs is shown in Appendix S1.

For each gametophyte, the proportions of homozygous and heterozygous loci ranged between 13.74 and $21.61 \%$ (mean of $17.04 \%$ ) and between 66.36 and $83.59 \%$ (mean of $77.12 \%$ ), respectively. The remainder were missing loci, representing an average $5.84 \%$ (Figure 1). An overwhelming number of heterozygous loci existed in all the gametophytes.

\section{Verification of Heterozygous SNPs}

The unmapped reads obtained from whole-genome resequencing were removed to filter out exogenous DNA contamination. However, because the reference genome was the result of a preliminary genome survey assembly, it was possible that exogenous DNA existed in the assembled 
TABLE 1 | Genotyping results of representative SNP loci in two parents and partial $F_{1}$ gametophytes using whole-genome resequencing technology.

\begin{tabular}{|c|c|c|c|c|c|c|c|c|c|c|c|}
\hline Chr & Position & Ref & Alt & $\$ 6$ & 89 & ZD10 & ZD20 & ZD30 & ZD40 & ZD50 & ZD60 \\
\hline Scaffold32 & $7259^{a}$ & $\mathrm{~T}$ & $A$ & W & W & W & W & W & W & W & W \\
\hline Scaffold19 & $12935^{b}$ & A & $\mathrm{G}$ & $A$ & $\mathrm{R}$ & $A$ & $\mathrm{R}$ & $R$ & $R$ & - & $R$ \\
\hline Scaffold42 & $123^{\mathrm{C}}$ & C & $\mathrm{T}$ & $\mathrm{T}$ & $\mathrm{T}$ & $\mathrm{T}$ & $\mathrm{T}$ & $T$ & $\mathrm{~T}$ & $\mathrm{~T}$ & $\mathrm{~T}$ \\
\hline Scaffold311 & $1271^{d}$ & $\mathrm{~T}$ & C & $\mathrm{T}$ & C & C & C & C & $\mathrm{C}$ & $\mathrm{C}$ & C \\
\hline Scaffold1438 & $26108^{\mathrm{e}}$ & $\mathrm{T}$ & $G$ & $\mathrm{~T}$ & $G$ & $\mathrm{~T}$ & $\mathrm{~T}$ & $\mathrm{~T}$ & $\mathrm{~K}$ & $\mathrm{~K}$ & G \\
\hline
\end{tabular}

Ref indicates the SNP genotype was in accordance with that of the reference genome sequence; Alt indicates that the SNP genotype was inconsistent with that of the reference genome sequence; $Z D X$ indicates No. $X F_{1}$ gametophyte; $W=A / T ; R=A / G ; K=G / T ;{ }^{a} T h e ~ S N P$ loci that had identical heterozygous genotypes in 62 gametophytes accounted for $48.07 \%$ of the total SNPs; ${ }^{b}$ The SNP loci that had a homozygous (or missing) genotype in one parent and a heterozygous genotype in the other accounted for $17.06 \%$ of the total SNPs; ${ }^{\circ}$ The SNP loci that had identical homozygous genotypes, but inconsistent with Ref, in the 62 gametophytes accounted for 7.43\% of the total SNPs; ${ }^{d}$ The SNP Ioci that had homozygous genotypes in the

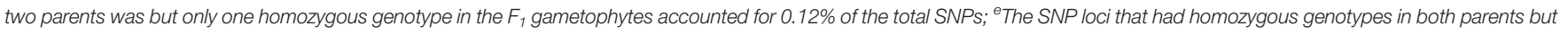
were heterozygous in some $F_{1}$ gametophytes accounted for $0.13 \%$ of the total SNPS.

reference genome. The GC content and average sequencing depth of the genomic data of Gp. lemaneiformis had been reported previously (Zhou et al., 2013) and are shown in Figure S3. The density points were concentrated in the 40$60 \%$ range, revealing no exogenous DNA contamination. For further verification, 6,755 SNPs that had identical heterozygous genotypes in the two parental gametophytes were annotated using BLASTN in NCBI. As shown in Table 2, 6,687 sequences were not annotated to any species, representing a proportion of $98.99 \%$. In total, 68 sequences were mapped to similar sequences in the NT database, with matched species from four classes, bacteria, fungi, red alga, and animal, accounting for only $1 \%$. The annotation results reflected the unlikelihood that the heterozygous SNPs were derived from exogenous DNA.

In this study, each gametophyte was cultivated in a separate bottle and the genomic DNA was extracted independently, which should eliminate the possibility of cross contamination. Moreover, a high proportion of heterozygous sites existed in each individual, as shown in Figure 2, which further indicated that the heterozygous SNPs did not result from cross contamination among certain individuals.
TABLE 2 | Annotation results of the sequences containing heterozygous SNP loci in Gracilariopsis lemaneiformis.

\begin{tabular}{llc}
\hline Species category & \multicolumn{1}{c}{ Species name } & Number of sequences \\
\hline Bacteria & Burkholderia multivorans & 5 \\
& Mixia osmundae & 9 \\
\multirow{5}{*}{ Fungus } & Scedosporium apiospermum & 1 \\
& total & $15(0.22 \%)$ \\
& Branchiostoma belcheri & 4 \\
& Saccharomyces cerevisiae & 4 \\
Rhodophyta & Naumovozyma dairenensis & 3 \\
& total & $11(0.16 \%)$ \\
& Chondrus crispus & 15 \\
& Furcellaria lumbricalis & 2 \\
& Gracilaria chorda & 2 \\
Zoology & Gracilaria gracilis & 3 \\
& Gracilaria vermiculophylla & 2 \\
& Gracilariopsis lemaneiformis & 3 \\
Unknown & total & $27(0.40 \%)$ \\
Total & Mus musculus & 1 \\
& Octodon degus & 5 \\
& Spodoptera frugiperda & 9 \\
& total & $15(0.22 \%)$ \\
& & $6758(98.99 \%)$
\end{tabular}

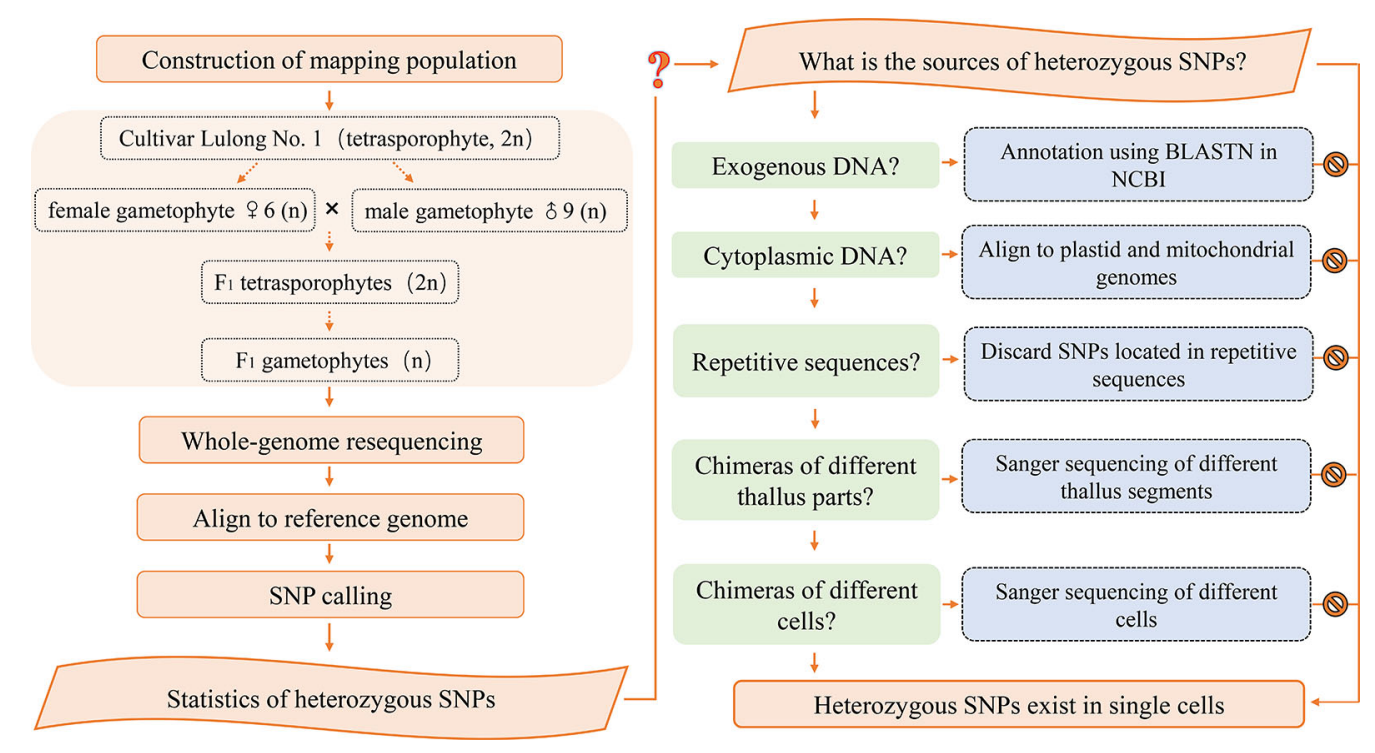

FIGURE 1 | Flowchart of the research methodology. 


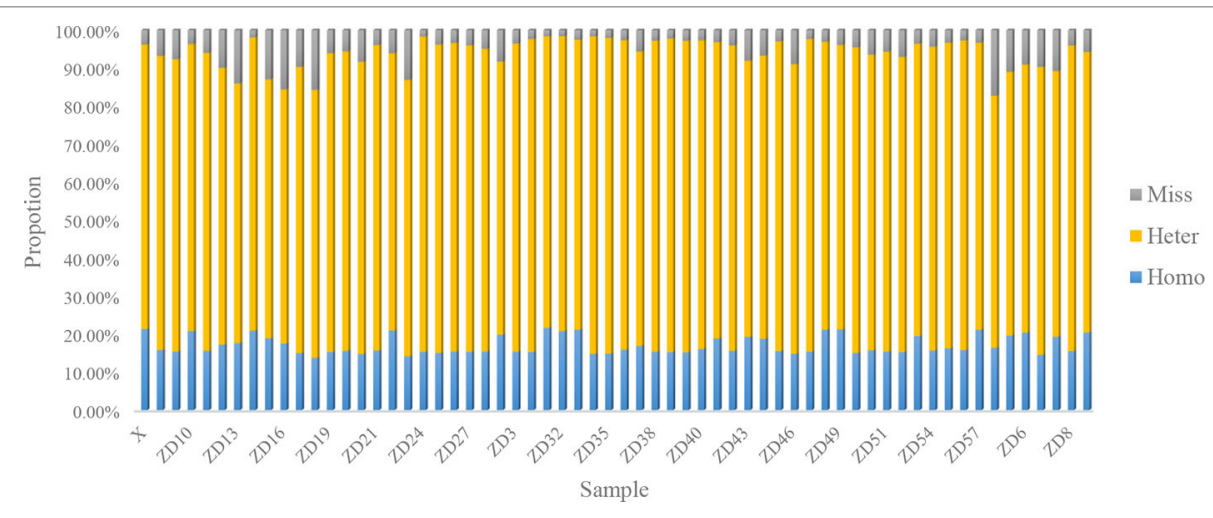

FIGURE 2 | Statistics of SNP type in parents and 60 gametophytes.

In total, 1,704 SNP loci that had homozygous (or missing) genotypes in one parent, but heterozygous genotypes in the other parent, were aligned to the complete plastid and mitochondrial sequences of Gp. lemaneiformis. However, none of the sequences containing SNPs were mapped to plastid or mitochondrial sequences, excluding the possibility that sequence contamination occurred from plastids or mitochondria.

Information regarding primers that were used to amplify SNP loci are listed in Table 3. Sanger sequencing results for three SNP loci in one $F_{1}$ gametophyte are displayed in Figure 3A. The genotyping results of the SNP locus scaffold1837_730 was A/G, and the Sanger sequencing of PCR products showed two peaks in this site, with peak A being higher than peak G. In addition, Sanger sequencing results of SNP loci scaffold7339_502 and scaffold7339_533 in this gametophyte also showed two peaks of SNPs that were consistent with the genotyping results obtained from whole-genome resequencing. To demonstrate the existence of the lower peak, monoclonal sequencing of the locus scaffold1837_730 was carried out in this gametophyte. In total, 54 monoclones were selected for sequencing. Of these, 53 monoclones had the single peak A and one monoclone had the single peak $G$, which verified the accuracy of the genotyping results.

\section{Excluding the Possibility of Chimerism}

All 22 segments that were cut from one gametophyte were amplified using primers for SNP locus scaffold1837_730. The Sanger sequencing results of each segment were completely coincident, showing two peaks, A and G, and even the height differences between peaks $A$ and $G$ were identical (Figure 3B).
Additionally, SNP loci scaffold7339_502 and scaffold7339_533 were detected in each part of the gametophyte. For each SNP locus, the Sanger sequencing results for each part were completely coincident and were consistent with the genotyping results derived from whole-genome resequencing, as shown in Figure 3B and Table 4.

\section{Detection of Heterozygous SNPs in a Single Cell}

Single cells were isolated and selected using glass capillary tube (Figure S4). Sanger sequencing results for three SNP loci (scaffold1837_730s, scaffold7339_502 and scaffold7339_533) in three cells from one $\mathrm{F}_{1}$ gametophyte are displayed in Figure 3C. The results were consistent with the genotyping results, as shown in Table 4. Thus, heterozygous SNP sites existed in single cells.

To eliminate background interference, high fidelity DNA polymerase was used to conduct the PCR amplification of three cells. Two SNP loci, scaffold376_4699 and scaffold2375_6885, which were located in genic regions, were further used to test single cells. Sanger sequencing results revealed that the two peaks of each SNP locus located in the genomic region still existed, which was consistent with the genotyping results of the whole-genome resequencing (Figure 3D). Thus, the SNP genotypes were shown to be heterozygous in individual cells.

\section{Inference That Heterozygous SNPs Exist in Haploid Gametophytes of Gp. lemaneiformis}

Based on our results, exogenous DNA contamination, cross contamination among individuals, plastid and mitochondrial

TABLE 3 | PCR primers used for Sanger sequencing.

\begin{tabular}{llcccccc}
\hline ID & \multicolumn{1}{c}{ Chr } & Position of SNP & Ref & Alt & Sense primer & Anti-sense primer & Product length (bp) \\
\hline P1 & scaffold1837 & 730 & A & G & TCCTCCTTACGGCGGTTGAGTT & TGGATACCGACTCGGACGAATACG \\
P2 & scaffold7339 & $502 / 533$ & A & T & AGCAGAAGACGGAGCGAACGATGA & AGTTGCAGCAATCCGGCCAATAGG & 301 \\
P3 & scaffold376 & 4699 & T & C & ATGTCTCGTACTTCGCTCCT & TACGGCGGTGAAACCGACTT \\
P4 & scaffold2375 & 6885 & G & A & CCTATGTCACCCGATTGTGT & ATGCAAGAGCGACTTGACGA & 485 \\
\end{tabular}




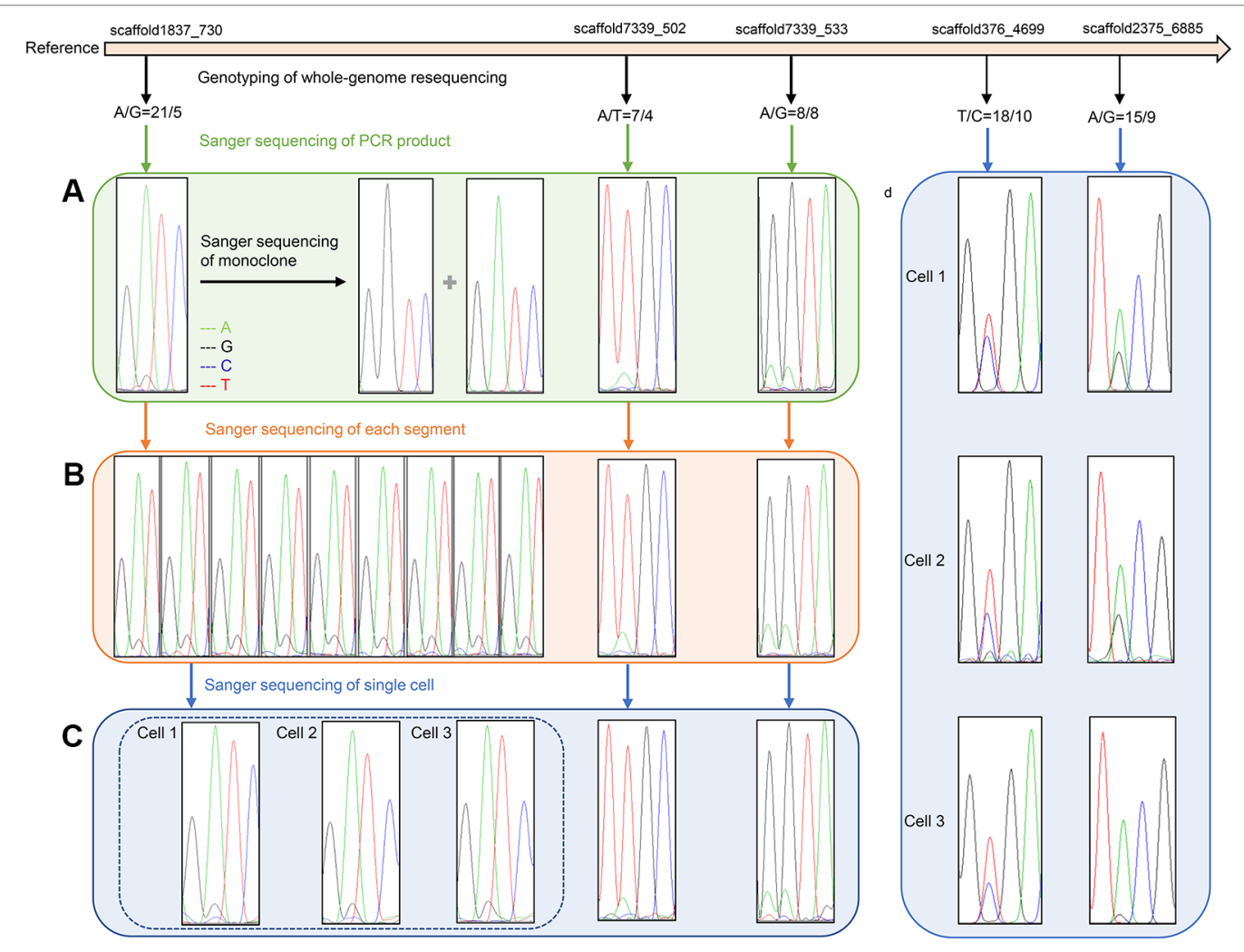

FIGURE 3 | Verification results of heterozygous SNPs (A) Sanger sequencing results of three SNP loci (scaffold1837_730, scaffold7339_502 and scaffold7339_533) in one $F_{1}$ gametophyte. Two peaks of each SNP cite are consistent with the genotyping results of whole-genome resequencing. Monoclonal sequencing results of the locus scaffold1837_730 show both single peak A and single peak G, which completely verified the accuracy of the genotyping results A/G. (B) Sanger sequencing results of three SNP loci in different segments of one $F_{1}$ gametophyte. The Sanger sequencing results of nine representative parts at locus scaffold1837_730 are completely coincident, showing two peak A and G, and are consistent with genotyping results AVG. (C) Sanger sequencing results of three SNP loci in three cells of one $F_{1}$ gametophytes. The Sanger sequencing results of three cells at locus scaffold1837_730 are completely coincident, showing two peaks A and G, and are consistent with the genotyping results A/G. (D) Sanger sequencing results of three cells at two SNP sites (scaffold376_4699 and scaffold2375_6885) located in gene regions. Two Sanger sequencing peaks of each SNP site located in gene region still existed, which are consistent with the genotyping results.

TABLE 4 | Genotyping results of 3 SNP loci in $F_{1}$ gametophytes (No.13) using whole-genome resequencing technology and Sanger sequencing results in different segments and single cells.

\begin{tabular}{|c|c|c|c|c|c|c|c|c|c|c|c|c|c|}
\hline ID of SNP loci & $F_{1}$ gametophyte (No.13) & \multicolumn{9}{|c|}{9 representative segments of $F_{1}$ gametophyte (No.13) } & \multicolumn{3}{|c|}{3 cells of $F_{1}$ gametophyte (No.13 } \\
\hline Scaffold 1837_730 & $A / G$ & $A / G$ & $A / G$ & $A / G$ & $A / G$ & $A / G$ & $A / G$ & $A / G$ & $A / G$ & $A / G$ & $A / G$ & $A / G$ & $A / G$ \\
\hline Scaffold 7339_502 & $\mathrm{A} T \mathrm{~T}$ & $\mathrm{~A} T \mathrm{~T}$ & $\mathrm{~A} / \mathrm{T}$ & $\mathrm{A} / \mathrm{T}$ & $\mathrm{A} / \mathrm{T}$ & $\mathrm{A} T \mathrm{~T}$ & $\mathrm{~A} / \mathrm{T}$ & $\mathrm{A} T \mathrm{~T}$ & $\mathrm{~A} / \mathrm{T}$ & $\mathrm{A} T \mathrm{~T}$ & $\mathrm{~A} T \mathrm{~T}$ & $\mathrm{~A} T \mathrm{~T}$ & $\mathrm{~A} / \mathrm{T}$ \\
\hline Scaffold 7339_533 & $A / G$ & $A / G$ & $A / G$ & $A / G$ & $A / G$ & $A / G$ & $A / G$ & $\mathrm{~A} / \mathrm{G}$ & $\mathrm{A} / \mathrm{G}$ & $A / G$ & $A / G$ & $A / G$ & $\mathrm{~A} / \mathrm{G}$ \\
\hline
\end{tabular}

sequences, chimeras of different thallus parts, or different cells, and repetitive sequences were excluded as being the source of heterozygous SNPs. However, the reference sequence we used to identify SNPs in this study, was a preliminary assembly version, with a scaffold N50 of $20 \mathrm{~kb}$ and a scaffold number of 125,685 . If a chromosomal rearrangement event, for instance, a duplication and deletion, occurred in the genome of Gp. lemaneiformis, the length of a scaffold might not cover the large area of duplication. Consequently, the unique scaffold may actually exist in multiple copies in the genome. This could explain why heterozygous SNPs existed in haploid gametophyte genomes.

\section{DISCUSSION}

In this study, whole-genome resequencing was conducted to develop SNP markers, which have been widely applied in many plants. Whole-genome resequencing of two parental lines Brassica oleracea L. C1184 and C1234 was performed to develop genome-wide SNP markers (Lee et al., 2015), and the sequencing data represented approximately 18 -fold genome coverage for both parental lines. After reference-based genomeguided mapping, 82.1 and $77.6 \%$ of reads from $\mathrm{C} 1184$ and $\mathrm{C} 1234$, respectively, were successfully aligned to the reference genome. 
As a result, a total of 674,521 SNPs was detected between C1184 and C1234. In Capsicum baccatum L. (Lee et al., 2016), the parental genomes were sequenced using the whole-genome resequencing method. After trimming the raw data, the parental sequences corresponded to approximately 3.1 and 9.6 times, respectively, the size of the reference genome. Then, $48.5 \%$ of the trimmed reads were successfully mapped to the reference sequences. Finally, 97,085 SNPs were selected as homozygous SNPs between the parents. Whole-genome resequencing was performed on $96 \mathrm{~F}_{6}$ recombinant inbred lines (RILs) of a cross between safflower Carthamus tinctorius L. and its wild progenitor C. palaestinus Eig (Bowers et al., 2016). Each RIL was sequenced to an average $2.26 \times$ coverage of the estimated genome, while the parents were sequenced to a greater depth. Sequence reads from the RILs were mapped to the reference genome, and 2,008,196 SNPs were identified.

In our result, the number of SNPs $(46,499)$ identified by whole-genome resequencing in Gp. lemaneiformis was smaller than in other plants, which might result from the small genome and population sizes. However, as $\mathrm{C}$-value paradox indicates that there is no absolute correlation between the size of a genome and evolutionary status of an organism. Although the genome size of Gp. lemaneiformis is only $97.02 \mathrm{Mb}$ (Zhou et al., 2013), its complexity level may be high.

The reference genome sequences selected in this study were from the first genome version, which consisted of 125,685 scaffolds with an N50 length of $20 \mathrm{~kb}$ (Zhou et al., 2013) and not from the second version, which consisted of 13,825 scaffolds with an N50 length of $30.59 \mathrm{~kb}$ (Sun et al., 2018). The main reason for the selection was that first genome sequencing was a wild female gametophyte, as our resequencing gametophyte, while in the second version, the material was the 981 strain, which is a diploid tetrasporophyte.

SNP markers were first developed in $\mathrm{F}_{1}$ gametophytes of $G p$. lemaneiformis using whole-genome resequencing technology, laying a foundation for construction of genetic linkage map and localization of quantitative trait locus. We further identified 2,386 SNPs which were located in 818 genes. For example, SNPs (scaffold2671_31163,31176,31189,31266) were located in the gene region of LEM_GLEAN_10003235 whose function was annotated as carotenoid isomerase, which is an important enzyme in synthesis pathway of carotenoids. Moreover, LEM_GLEAN_0001204, the gene encoding phosphoglucose isomerase in metabolic pathways of agar, contains one SNP locus (scaffold1981_ 16188). These SNPs could be potentially used as genetic markers for the selection and provided the theoretical basis of molecular marker assisted breeding

An overwhelming number of heterozygous SNP loci were verified to exist in the 62 gametophytes, which might result from chromosomal duplication events in the genome of Gp. lemaneiformis. There are several kinds of chromosomal rearrangement, including the duplication or deletion of a chromosomal segment, the inversion of a segment and the translocation of segments between non-homologous chromosomes (Rieseberg 2001). Each chromosomal rearrangement event can be caused by the breakage of DNA double-helices at two different locations, followed by rejoining or by crossing-over between repetitive DNA (Griffiths et al., 1999). Chromosomal rearrangements in the genome can produce duplicated genes, which may differ in DNA sequence, gene structure and even function. One genetic effect of duplication is that the gene expression may be enhanced as the gene copies increase in number.

A chromosome level of assembly is required to verify the source of the heterozygous SNPs. This study promoted the assembly of a Gp. lemaneiformis genomic map of using single molecule real-time sequencing (SMRT), or high-throughput chromosome conformation capture. SMRT sequencing can produce considerably longer and highly accurate reads from single unamplified molecules. The average read length of PacBio RS II platform is about $15 \mathrm{~kb}$. The long SMRT sequencing reads will span complex repeats and missing bases, which will substantially improve genome complete assembly. A complete genome has the potential to facilitate genome-assisted breeding and will be useful to investigate genomic patterns in the evolution of marine algae.

\section{DATA AVAILABILITY STATEMENT}

The datasets generated for this study can be found in the Short Read Archive (SRA) database with Project number PRJNA574029, under the accession number SRP223151 (SRR10177194 - SRR10177255).

\section{AUTHOR CONTRIBUTIONS}

$\mathrm{ZS}$ and $\mathrm{YH}$ contributed conception and design of the study. $\mathrm{YH}$, WZho and JW constructed the mapping population. $\mathrm{YH}$ performed the experiments. $\mathrm{YH}$ and $\mathrm{ZS}$ analyzed the data. MJ, HC, JZ, WZha, XF, YL, BX and PG contributed reagents/ materials/analysis tools. $\mathrm{YH}$ wrote the manuscript and $\mathrm{ZS}$ made revision. All authors read and approved the submitted version.

\section{FUNDING}

This work was supported by the China Agriculture Research System (CARS-50), the National Natural Science Foundation of China (No. 31372529) and the Fundamental Research Funds for the Central Universities (No. 201762016).

\section{SUPPLEMENTARY MATERIAL}

The Supplementary Material for this article can be found online at: https://www.frontiersin.org/articles/10.3389/fgene.2019. 01256/full\#supplementary-material 


\section{REFERENCES}

Baird, N. A., Etter, P. D., Atwood, T. S., Currey, M., Shiver, A. L., Lewis, Z. A., et al. (2008). Rapid SNP discovery and genetic mapping using sequenced RAD markers. PloS One 3, e3376. doi: 10.1371/journal.pone.0003376

Berger, J., Suzuki, T., Senti, K., Stubbs, J., Schaffner, G., and Dickson, B. J. (2001). Genetic mapping with SNP markers in Drosophila. Nat. Genet. 29, 475-481. doi: $10.1038 /$ ng773

Bowers, J. E., Pearl, S. A., and Burke, J. M. (2016). Genetic mapping of millions of SNPs in safflower (Carthamus tinctorius L.) via whole genome resequencing. G3: Genes Genom. Genet. 6, 2203-2211. doi: 10.1534/g3.115.026690

Cao, J., Schneeberger, K., Ossowski, S., Gunther, T., Bender, S., Fitz, J., et al. (2011). Whole-genome sequencing of multiple Arabidopsis thaliana populations. Nat. Genet. 43, 956-963. doi: 10.1038/ng.911

Chen, D., Ahlford, A., Schnorrer, F., Kalchhauser, I., Fellner, M., Viragh, E., et al. (2008). High-resolution, high-throughput SNP mapping in Drosophila melanogaster. Nat. Methods 5, 323-329. doi: 10.1038/nmeth.1191

Chopin, T., Buschmann, A. H., Halling, C., Troell, M., Kautsky, N., Neori, A., et al. (2001). Integrating seaweeds into marine aquaculture systems: a key toward sustainability. J. Phycol. 37, 975-986. doi: 10.1046/j.1529-8817.2001.01137.x

Ding, H. Y., Sui, Z. H., Zhong, J., Zhou, W., and Wang, Z. X. (2012). Analysis and comparison on genetic diversity of haploid and diploid Gracilaria lemaneiformis populations from different places of Qingdao by AFLP. Period Ocean Univ. China 42, 99-105. doi: 10.1007/s11783-011-0280-z

Du, Q. W., Bi, G. Q., Mao, Y. X., and Sui, Z. H. (2016). The complete chloroplast genome of Gracilariopsis lemaneiformis (Rhodophyta) gives new insight into the evolution of family Gracilariaceae. J. Phycol. 52, 441-450. doi: 10.1111/ jpy. 12406

Engel, C., Aberg, P., Gaggiotti, O. E., Destombe, C., and Valero, M. (2010). Population dynamics and stage structure in a haploid-diploid red seaweed, Gracilaria gracilis. J. Ecol. 89, 436-450. doi: 10.1046/j.1365-2745.2001.00567.x

Fei, X. G. (2004). Solving the coastal eutrophication problem by large scale seaweed cultivation. Hydrobiologia 512, 145-151. doi: 10.1023/b: hydr.0000020320.68331.ce

Fu, F., Sui, Z. H., Zhou, W., Wang, J. G., Chang, L. P., and Ci, S. F. (2014). UVirradiation mutation of tetraspores of Gracilariopsis lemaneiformis and screening of thermotolerant strains. J. Appl. Phycol. 26, 647-656. doi: 10.1007/s10811-013-0087-3

Fu, B., Liu, H., Yu, X., and Tong, J. (2016). A high-density genetic map and growth related QTL mapping in bighead carp (Hypophthalmichthys nobilis). Sci. Rep. 6, 28679. doi: $10.1038 /$ srep28679

Griffiths, A. J. F., Gelbart, W. M., Miller, J. H., and Lewontin., R. C. (1999). Modern Genetic Analysis (New York: W. H. Freeman), p. 319 pp.

Guzmán-Urióstegui, A., García-Jiménez, P., Marián, F., Robledo, D., and Robaina, R. (2002). Polyamines influence maturation in reproductive structures of Gracilaria cornea (Gracilariales, Rhodophyta). J. Phycol. 38, 1169-1175. doi: 10.1046/j.15298817.2002.01202.x

Hu, Y. Y., Sui, Z. H., Zhou, W., Wang, J. G., Chang, L. P., Guo, W. H., et al. (2018). Development of genomic simple sequence repeat markers and genetic diversity analysis of Gracilariopsis lemaneiformis (Rhodophyta). J. Appl. Phycol. 30, 707-716. doi: 10.1007/s10811-017-1237-9

Hu, Y. Y., Du, Q. W., Mi, P., Shang, E. L., and Sui, Z. H. (2019). Gene cloning and expression regulation in the pathway of agar and floridean starch synthesis of Gracilariopsis lemaneiformis (Rhodophyta). J. Appl. Phycol. 31, 1889-1896. doi: 10.1007/s10811-018-1690-0

Huang, X., Feng, Q., Qian, Q., Zhao, Q., Wang, L., Wang, A., et al. (2009). Highthroughput genotyping by whole-genome resequencing. Genome Res. 19, 1068-1076. doi: 10.1101/gr.089516.108

Kerstens, H. H., Crooijmans, R. P., Veenendaal, A., Dibbits, B. W., Chin-A-Woeng, T. F., Dunnen, J. T. D., et al. (2009). Large scale single nucleotide polymorphism discovery in unsequenced genomes using second generation high throughput sequencing technology: applied to turkey. BMC Genomics 10, 479. doi: 10.1186/ 1471-2164-10-479

Lam, H. M., Xu, X., Liu, X., Chen, W. B., Yang, G. H., Wong, F. L., et al. (2010). Resequencing of 31 wild and cultivated soybean genomes identifies patterns of genetic diversity and selection. Nat. Genet. 42, 1053-1059. doi: 10.1038/ng.715
Lee, J., Izzah, N. K., Jayakodi, M., Perumal, S., Joh, H. J., Lee, H. J., et al. (2015). Genome-wide SNP identification and QTL mapping for black rot resistance in cabbage. BMC Plant Biol. 15, 32. doi: 10.1186/s12870-015-0424-6

Lee, Y. R., Yoon, J. B., and Lee, J. (2016). A SNP-based genetic linkage map of Capsicum baccatum and its comparison to the Capsicum annuum reference physical map. Mol. Breed. 36, 61. doi: 10.1007/s11032-016-0485-8

Li, C. L., Bai, G. H., Carver, B. F., Chao, S. M., and Wang, Z. H. (2015). Single nucleotide polymorphism markers linked to QTL for wheat yield traits. Euphytica 206, 89-101. doi: 10.1007/s10681-015-1475-3

Liu, Y. T., Zhang, X., Sun, H. Y., Yang, Q., Zang, X. N., Zhang, X. C., et al. (2016). Cloning and transcription analysis of six members of the calmodulin family in Gracilaria lemaneiformis under heat shock. J. Appl. Phycol. 28, 643-651. doi: 10.1007/s10811-015-0575-8

Liu, Y. T., Sun, H. Y., Ding, Y., Zang, X. N., and Zhang, X. C. (2018). A novel heat shock protein from Gracilariopsis lemaneiformis: gene cloning and transcription analysis in response to heat stress. J. Appl. Phycol. 30, 36233631. doi: 10.1007/s10811-018-1494-2

Lu, H., Xie, H., Gong, Y., Wang, Q., and Yang, Y. (2011). Secondary metabolites from the seaweed Gracilaria lemaneiformis and their allelopathic effects on Skeletonema costatum. Biochem. Syst. Ecol. 39, 397-400. doi: 10.1016/ j.bse. 2011.05 .015

Lu, J. J., Liu, Y. Y., Xu, J., Mei, Z. W., Ziwei, M., Shi, Y. J., et al. (2018). High-density genetic map construction and stem total polysaccharide content-related qtl exploration for chinese endemic Dendrobium (Orchidaceae). Front. Plant Sci. 9, 398. doi: 10.3389/fpls.2018.00398

Mchugh, D. J. (1991). Worldwide distribution of commercial resources of seaweeds including Gelidium. Hydrobiologia 221, 19-29. doi: 10.1007/ BF00028359

Neori, A., Chopin, T., Troell, M., Buschmann, A. H., Kraemer, G. P., Halling, C., et al. (2004). Integrated aquaculture: rationale, evolution and state of the art emphasizing seaweed biofiltration in modern mariculture. Aquaculture 231, 361-391. doi: 10.1016/j.aquaculture.2003.11.015

Pang, Q., Sui, Z., Kang, K. H., Kong, F., and Zhang, X. (2010). Application of SSR and AFLP to the analysis of genetic diversity in Gracilariopsis lemaneiformis (Rhodophyta). J. Appl. Phycol. 22, 607-612. doi: 10.1007/s10811-009-9500-3

Plastino, E. M., and de Oliveira, F. E. C. (1988). Deviations in the life-history of Gracilaria sp. (Rhodophyta, Gigartinales), from Coquimbo, Chile, under different culture conditions. Hydrobiologia 164, 67-74. doi: 10.1007/ bf00014350

Poland, J., Brown, P. J., Sorrells, M. E., and Jannink, J. (2012). Development of high-density genetic maps for barley and wheat using a novel two-enzyme genotyping-by-sequencing approach. PloS One 7, e32253. doi: 10.1371/ journal.pone. 0032253

Raman, D., George, F., Yves, D., Curtis, P., Laroche, A., Eudes, F., et al. (2018). High density single nucleotide polymorphism (SNP) mapping and quantitative trait loci (QTL) analysis in a biparental spring triticale population localized major and minor effect fusarium head blight resistance and associated traits QTL. Genes 9, 19. doi: 10.3390/genes9010019

Ribaut, J. M., and Hoisington, D. A. (1998). Marker-assisted selection: new tools and strategies. Trend Plant Sci. 3, 236-239. doi: 10.1016/s1360-1385(98)01240-0

Rieseberg, L. (2001). Chromosomal rearrangements and speciation. Trends Ecol. Evol. 16, 351-358. doi: 10.1016/S0169-5347(01)02187-5

Santelices, B., and Doty, M. S. (1989). A review of Gacilaria farming. Aquaculture 78, 95-133. doi: 10.1016/0044-8486(89)90026-4

Stickney, H. L., Schmutz JWoods, I. G., Holtzer, C. C., Dickson, M. C., Kelly, P. D., Myers, R. M., et al. (2002). Rapid mapping of zebrafish mutations with SNPs and oligonucleotide microarrays. Genome Res. 12, 1929-1934. doi: 10.1101/ gr.777302

Sun, X., Wu, J., Wang, G., Kang, Y., Ooi, H. S., Shen, T., et al. (2018). Genomic analyses of unique carbohydrate and phytohormone metabolism in the macroalga Gracilariopsis lemaneiformis (Rhodophyta). BMC Plant Biol. 18, 94. doi: 10.1186/s12870-018-1309-2

Wang, W. J., Wang, Z. Q., Lin, X. Z., and Xu, P. (2007). Characterization of Gracilaria lemaneiformis Bory (Gracilariaceae, Rhodophyta) cultivars in China using the total soluble proteins and RAPD analysis. Bot. Mar. 50, 177-184. doi: 10.1515/bot.2007.021 
Wang, S., Meyer, E., Mckay, J. K., and Matz, M. V. (2012). 2b-RAD: a simple and flexible method for genome-wide genotyping. Nat. Methods 9, 808-810. doi: 10.1038/nmeth.2023

Wicks, S. R., Yeh, R. T., Gish, W. R., Waterston, R. H., and Plasterk, R. H. A. (2001). Rapid gene mapping in Caenorhabditis elegans using a high density polymorphism map. Nat. Genet. 28, 160-164. doi: 10.1038/88878

Xia, Q., Guo, Y., Zhang, Z., Li, D., Xuan, Z., Li, Z., et al. (2009). Complete resequencing of 40 genomes reveals domestication events and genes in silkworm (Bombyx). Science 326, 433-436. doi: 10.1126/science.1176620

Yamamoto, H., and Sasaki, J. (1988). Interfertility between so-called Gracilaria verrucosa (Huds.) Papenfuss and G. vermiculophylla (Ohmi) Papenfuss in Japan. Bull. Fac. Fish. Hokkaido Univ. 39, 1-3.

Zhang, X. C., and van der Meer, J. P. (2011). A genetic study on Gracilaria sjoestedtii. Can. J. Bot. 66, 2022-2026. doi: 10.1139/b88-276

Zhang, L., Wang, X. M., Qian, H., Chi, S., Liu, C., and Liu, T. (2012). Complete sequences of the mitochondrial DNA of the wild Gracilariopsis lemaneiformis and two mutagenic cultivated breeds (Gracilariaceae, Rhodophyta). PloS One 7, e40241. doi: 10.1371/journal.pone.0040241
Zhou, W., Hu, Y. Y., Sui, Z. H., Fu, F., Wang, J. G., Chang, L. P., et al. (2013). Genome Survey Sequencing and Genetic Background Characterization of Gracilariopsis lemaneiformis (Rhodophyta) Based on NextGeneration Sequencing. PloS One 8, e69909. doi: 10.1371/journal. pone.0069909

Conflict of Interest: The authors declare that the research was conducted in the absence of any commercial or financial relationships that could be construed as a potential conflict of interest.

Copyright (c) $2019 \mathrm{Hu}$, Sui, Zhou, Wang, Jiang, Chen, Zhang, Zhang, Feng, Lei, Xiao and Guo. This is an open-access article distributed under the terms of the Creative Commons Attribution License (CC BY). The use, distribution or reproduction in other forums is permitted, provided the original author(s) and the copyright owner(s) are credited and that the original publication in this journal is cited, in accordance with accepted academic practice. No use, distribution or reproduction is permitted which does not comply with these terms. 